\title{
Blood Gene Expression Risk Profiles and Interstitial Lung Abnormalities: COPDGene and ECLIPSE cohort studies
}

Matthew Moll ${ }^{1,2,3^{*}}$, Brian D. Hobbs ${ }^{1,2,3^{*}}$, Aravind Menon ${ }^{2,3}$, Auyon J. Ghosh ${ }^{1,2,3}$, Rachel K. Putman ${ }^{2,3}$, Takuya Hino ${ }^{3,4}$, Akinori Hata ${ }^{3,4}$, Edwin K. Silverman ${ }^{1,2,3}$, John Quackenbush ${ }^{1,5}$, Peter J. Castaldi ${ }^{1,3,6}$, Craig P. Hersh ${ }^{1,2,3}$, Michael J. McGeachie ${ }^{1}$, Don D. Sin ${ }^{7}$, Ruth Tal-Singer ${ }^{8}$, Mizuki Nishino ${ }^{3,4}$, Hiroto Hatabu ${ }^{3,4}$, Gary M. Hunninghake ${ }^{2,3 \dagger}$, Michael H. Cho ${ }^{1,2,3 \dagger}$

Affiliations:

1 Channing Division for Network Medicine, Brigham and Women’s Hospital, Boston, MA 02115, USA

2 Division of Pulmonary and Critical Care Medicine, Brigham and Women's Hospital, Boston, MA 02115, USA

3 Harvard Medical School, Boston, MA 02115, USA

4 Center for Pulmonary Functional Imaging, Department of Radiology, Brigham and Women's Hospital, Boston, MA 02115, USA

5 Department of Biostatistics, Harvard T.H. Chan School of Public Health, Boston, MA 02115, USA

6 Division of General Internal Medicine and Primary Care, Department of Medicine, Brigham and Women's Hospital, Boston MA, 02115

7 Centre for Heart Lung Innovation, St. Paul's Hospital, and Department of Medicine (Respiratory Division), University of British Columbia, Vancouver, BC, Canada.

8 COPD Foundation, Washington D.C., USA

$*$ = Equal contributions and co-first authors

${ }^{\dagger}=$ Jointly supervised and co-senior authors

Correspondence:

Michael H. Cho, M.D., M.P.H.

181 Longwood Ave, 4th floor

Boston, MA 02115

Email: $\underline{\text { remhc@channing.harvard.edu }}$ 
Funding:

MM and AJG are supported by T32HL007427.

BDH is supported by NIH K08HL136928 and U01 HL089856.

CPH is supported by NIH R01HL130512, R01HL157879 and P01HL114501.

PJC is supported by NIH R01HL124233 and R01HL147326.

EKS is supported by NIH R01 HL137927, R01 HL147148, U01 HL089856, R01 HL133135, R01 HL152728, P01 HL132825, and P01 HL114501.

HH is supported by NIH R01CA203636, 5U01CA209414, NIH/NHLBI 2R01HL111024, NIH R01HL135142, and NIH/NHLBI

1R01HL130974.

MN is supported by R01CA203636, U01CA209414, R01HL111024.

GMH is supported by NIH R01 HL111024, R01 HL130974, and R0135142.

MHC is supported by NIH R01HL137927, R01HL135142, HL147148, and HL089856.

The COPDGene project described was supported by Award Number U01 HL089897 and Award Number U01 HL089856 from the National Heart, Lung, and Blood Institute. The content is solely the responsibility of the authors and does not necessarily represent the official views of the National Heart, Lung, and Blood Institute or the National Institutes of Health. COPDGene is also supported by the COPD Foundation through contributions made to an Industry Advisory Board that has included AstraZeneca, Bayer Pharmaceuticals, Boehringer Ingelheim, Genentech, GlaxoSmithKline, Novartis, Pfizer, and Sunovion. The ECLIPSE study (NCT00292552; GSK code SCO104960) was funded by GlaxoSmithKline.

\section{Disclosures:}

EKS received grant support from GlaxoSmithKline and Bayer. CPH reports grant support from Boehringer-Ingelheim, Novartis, Bayer and Vertex, outside of this study. MN has received research grants to the institution from AstraZeneca, Daiichi Sankyo, Canon Medical Systems, and has served as a consultant to AstraZeneca, and Daiichi Sankyo. PJC has received grant support from GlaxoSmithKline and Bayer and consulting fees from GlaxoSmithKline and Novartis. RTS was employed by GlaxoSmithKline until 2019 and is a shareholder of GlaxoSmithKline stock; RTS received consulting fees from Immunomet, ENA Respiratory, Teva, and Vocalis Health; she is currently a Board Member of ENA Respiratory on behalf of the COPD Foundation. JQ is on the scientific advisory boards of Caris Life Sciences and Renalytix. HH received grants from Canon Medical Systems Inc and Konica-Minolta Inc. and served as consultant for Mitsubishi Chemical Co and Canon Medical Systems Inc outside of this study. GMH has performed consulting work for Boehringer-Ingelheim, and the Gerson Lehrman Group within the last 3 years. MHC has received grant support from GlaxoSmithKline and Bayer, consulting fees from Genentech and AstraZeneca, and speaking fees from Illumina. 
Author contributions:

Study Design: Matthew Moll, Brian D. Hobbs, Gary M. Hunninghake, Michael H. Cho.

Acquisition, analysis, or interpretation of the data: Matthew Moll, Auyon Ghosh, Don D. Sin, Peter J. Castaldi, Brian D. Hobbs, Edwin K. Silverman, John Quackenbush, Hiroto Hatabu, Takuya Hino, Akinori Hata, Mizuki Nishino, Gary M. Hunninghake, Michael H. Cho.

Critical revision of the manuscript for important intellectual content: All authors

Statistical analysis: Matthew Moll, Brian D. Hobbs, Auyon Ghosh, Gary M. Hunninghake, Michael H. Cho.

Obtained funding: Edwin K. Silverman, Gary M. Hunninghake, Michael H. Cho.

Word counts:

Abstract: 250

Manuscript: 4409

\section{Key messages}

What is the key question?

Interstitial lung abnormalities (ILA) are radiologic findings that may progress to idiopathic pulmonary fibrosis (IPF). Do blood gene expression profiles that predict IPF mortality also associate with ILA?

What is the bottom line?

An ILA gene expression score, derived from IPF mortality-associated genes, was associated with ILA and all-cause mortality. This score identified genes with concordant and discordant effects on IPF mortality and ILA. Our results suggest shared, and unique biologic processes, amongst those with ILA, IPF, aging, and death.

Why read on?

Our results lend insight into how gene expression profiles and biological pathways associated with IPF prognosis relate to ILA and allcause mortality 


\section{Abstract}

Rationale: Interstitial lung abnormalities (ILA) are radiologic findings that may progress to idiopathic pulmonary fibrosis (IPF). Blood gene expression profiles can predict IPF mortality, but whether these same genes associate with ILA and ILA outcomes is unknown.

Objectives: To evaluate if a previously described blood gene expression profile associated with IPF mortality is associated with ILA and all-cause mortality.

Methods: In COPDGene and ECLIPSE study participants with visual scoring of ILA and gene expression data, we evaluated the association of a previously described IPF mortality score with ILA and mortality. We also trained a new ILA score, derived using genes from the IPF score, in a subset of COPDGene. We tested the association with ILA and mortality on the remainder of COPDGene and ECLIPSE.

Measurements and Main Results: In 1,469 COPDGene (training n=734; testing $n=735$ ) and 571 ECLIPSE participants, the IPF score was not associated with ILA or mortality. However, an ILA score derived from IPF score genes was associated with ILA (metaanalysis of test datasets OR 1.4 [95\% CI: 1.2-1.6]) and mortality (HR 1.25 [95\% CI: 1.12-1.41]). Six of the 11 genes in the ILA score had discordant directions of effects compared to the IPF score. The ILA score partially mediated the effects of age on mortality (11.8\% proportion mediated).

Conclusions: An ILA gene expression score, derived from IPF mortality-associated genes, identified genes with concordant and discordant effects on IPF mortality and ILA. These results suggest shared, and unique biologic processes, amongst those with ILA, IPF, aging, and death. 


\section{Introduction}

Interstitial lung abnormalities (ILA) are specific radiologic findings detected on computed tomography (CT) scans ${ }^{1-3}$ and, in some instances, may represent, or progress to, pulmonary fibrosis ${ }^{4,5}$. Both ILA and idiopathic pulmonary fibrosis (IPF) are associated with pulmonary symptoms ${ }^{2,6}$, diminished lung function ${ }^{1,2,4,5,7,8}$, and mortality ${ }^{9}$. Despite these clinical similarities, genetic analyses reveal that these entities have both overlapping and distinct genetic risk alleles ${ }^{10,11}$. Taken together, the clinical and genetic evidence suggests that ILA and IPF possess both shared and unique pathobiology.

The risk of IPF and IPF mortality are not due to genetic variants alone ${ }^{12}$. Gene expression data can reflect the combination of both genetic variation and environmental factors that contribute to IPF pathogenesis (e.g. cigarette smoking) $)^{13}$. Herazo-Maya et al. ${ }^{14}$ used blood microarray data to develop a 52-gene IPF risk score that predicted mortality in multiple IPF cohorts ${ }^{15}$. Whether this IPF mortality risk score, or the specific genes in this risk score, are associated with ILA or ILA mortality in current and former smokers is not known. A peripheral blood signature that predicted ILA and mortality in ILA could be important for identifying early disease and those at risk for worse outcomes. In addition, shared gene expression features of IPF progression and ILA risk would highlight important biologic processes associated with the spectrum of interstitial lung disease from precursor lesions to irreversible fibrosis to death.

Therefore, we hypothesized that the IPF risk score would be associated with ILA and ILA-associated mortality, and that this risk would be driven by a subset of the genes in the IPF mortality risk score; this subset of genes may also lend insight into the biologic mechanisms relating ILA to fibrosis and death. To test this hypothesis, we utilized participants with visual ILA scoring and peripheral blood RNA-seq data from the Genetic Epidemiology of COPD (COPDGene) study ${ }^{16}$, and participants with peripheral blood microarray gene expression data from the Evaluation of COPD Longitudinally to Identify Predictive Surrogate End-points (ECLIPSE) study ${ }^{17}$. We first assessed the association of the previously developed IPF risk score with ILA and time-to-death. As we hypothesized that ILA risk would be captured by a subset of these IPF risk score genes, we also created a gene expression risk score, based on these same genes, optimized for the outcome of ILA. We assessed the performance and differences between the scores and used overrepresentation analyses to gain insight into the biologic processes most relevant to ILA and all-cause mortality.

\section{Methods}

Study populations

All study participants provided written informed consent. Each study center obtained institutional review board approval. 


\section{COPDGene}

We included participants from the Genetic Epidemiology of COPD (COPDGene) study ${ }^{16}$ who had a 5-year follow up visit with blood RNA-sequencing (RNA-seq) data and computed tomography (CT) scans that were assessed for the presence ILA ${ }^{9}$. Details of the COPDGene study have been previously described ${ }^{16}$. Briefly, COPDGene is a prospective cohort study of non-Hispanic white (NHW) and African American (AA) smokers ( $\geq 10$ pack-years of smoking), aged 45-80 years at study initiation, with and without COPD. The study was originally conceived of as a case-control study and has been extended into a longitudinal study with 5- and 10-year follow up visits. Whole blood samples, as well as anthropometric, spirometry, and CT imaging data were collected at each visit.

\section{ECLIPSE}

We included Evaluation of COPD Longitudinally to Identify Predictive Surrogate End-points (ECLIPSE) study ${ }^{17}$ participants with microarray gene expression data and chest CT scans that were assessed for the presence of ILA ${ }^{9}$. ECLIPSE participants were smokers ( $\geq 10$ pack-years of smoking) aged 45-75 years at study enrollment. Baseline questionnaire, spirometry, CT imaging and blood samples were collected. In ECLIPSE, COPD participants, but not controls, were followed longitudinally for 3 years.

\section{ILA phenotyping}

In both COPDGene and ECLIPSE, thoracic CT scans were assessed for ILA using a sequential method by three readers as previously described $^{10,11}$. The definition of ILA included in this manuscript conforms to the updated definition utilized by the Fleischner society $^{3,18-20}$.

Preparation of gene expression data

\section{RNA sequencing}

COPDGene whole blood RNA-seq data was available at the 5-year follow up visit, and data generation was previously described ${ }^{13}$. Briefly, PAXgene Blood RNA tubes were used to collect whole blood samples, and the Qiagen PreAnalytiX PAXgene Blood miRNA Kit (Qiagen, Valencia, CA) was used to extract total RNA. Samples with concentrations > $25 \mathrm{ug} / \mathrm{uL}$ and RNA integrity number (RIN) > 6 were eligible for sequencing. TruSeq Stranded Total RNA with Ribo-Zero Globin kit (Illumina, Inc., San Diego, CA) were used for globin-reduction and cDNA library preparation. The Illumina HiSeq 2500 sequencer was used to generate 75 bp reads with a mean of 20 million reads per sample. Skewer ${ }^{21}$ was used to trim TruSeq adapter sequences.

Additional quality control was performed using FASTQC (https://www.bioinformatics.babraham.ac.uk/projects/fastqc/) and RNA$\mathrm{SeQC}^{22}$. Reads were aligned to the human GRCh38 reference genome using STAR $2.5^{23}$. Count data were adjusted for library depth and batch effects were removed using the limma removeBatchEffects function ${ }^{24}$. 
Microarray gene expression data

ECLIPSE whole blood microarray data were available at the initial enrollment visit. Details regarding microarray data collection and processing were previously published ${ }^{25}$. Total RNA was extracted using PAXgene Blood miRNA kits and hybridized to the Affymetrix Human Gene 1.1 ST array. Quality control metrics were implemented using the Bioconductor oligo ${ }^{26}$ and RMA Express $^{27}$ packages. The Factor Analysis for Robust Microarray Summarization ${ }^{28}$ package was utilized for background correction and normalization. Batch effects were removed using the limma removeBatchEffects function ${ }^{24}$. As some gene transcripts were represented by multiple probes, we chose the probe with the greatest interquartile range, as previously described ${ }^{14,15}$.

We then took additional steps to facilitate comparability across RNA-seq and microarray data technologies: (1) we limited transcripts to those present in both data sets based on HGNC symbols; (2) we scaled and centered all gene expression data to have a mean of zero and a standard deviation of 1 .

\section{Statistical analyses}

Overview of study design

COPDGene was used as a discovery and testing cohort, and ECLIPSE was used for independent replication (Figure 1). In COPDGene, risk score training was performed in half the participants with the other half of the participants used for risk score testing. We assessed the association of three transcriptome-based risk scores (see Predictors) with outcomes (see Outcomes) in the COPDGene testing sample. We attempted to replicate the risk score associations in ECLIPSE.

\section{Predictors}

We calculated the Herazo-Maya et al. ${ }^{14,15}$ IPF prognosis signature using transcriptomic data from COPDGene and ECLIPSE and applied this risk signature to the prediction of ILA and mortality in these cohorts. Two transcripts were not available in our cohorts (C2ORF27A and SNHG1), resulting in 50 genes available for testing. Briefly, calculating this risk score involves calculating the proportion of up- and down-regulated genes, summing the normalized expression values, calculating the product between the summed normalized expression values and proportion of decreased and increased genes, and comparing the up- and down- scores to the median for the population. If both the up- and down- scores are higher than the median of the population in which the score is being calculated, then the person is considered to be at high IPF mortality risk ( $1=$ high IPF mortality risk, $0=$ low IPF mortality risk).

To test the performance of these same genes using an alternative method, we developed a risk score optimized to the outcome of ILA by taking the following steps: 
(1) We used the 50 available transcripts as inputs to construct a penalized regression (Least Absolute Shrinkage and Selection Operator (LASSO)) model in the COPDGene training set optimized to the outcome of ILA. LASSO regression shrinks coefficients toward zero to provide feature selection and minimizes collinearity amongst predictors. We tuned models within the COPDGene training set using 5-fold cross-validation, optimizing the area-under-the-receiver-operating-curve (AUC) on the left-out fold.

(2) We used this penalized regression model (weights of gene transcripts) to calculate the log odds for ILA for each individual in the COPDGene testing sample and ECLIPSE.

(3) We scaled and centered the risk scores within the COPDGene testing and ECLIPSE datasets separately.

Thus, we evaluated two main predictors in this study: (1) the original IPF mortality risk score, and (2) a re-weighted risk score optimized to ILA as an outcome (hereafter, "ILA score [IPF transcripts]"). Results were presented as 1 standard deviation increases in risk scores. Each risk score was tested in the COPDGene testing set and the ECLIPSE replication cohort. We additionally compared the direction of effects of genes in these scores. We range-standardized both risk scores and plotted histograms of predicted probabilities for each respective outcome in the COPDGene testing set.

As an additional analysis, we created an ILA-optimized risk score using genome-wide transcripts (hereafter, "ILA score [all transcripts]"). Prior to training the ILA score [all transcripts], we limited our analyses to highly expressed transcripts (>1 count per million in $99 \%$ of samples), resulting in 9,100 transcripts. These 9,100 transcripts were used as inputs into LASSO regression. The model was tuned within the COPDGene training set using 10-fold cross-validation, minimizing misclassification error on the left-out fold. We then calculated and standardized the risk scores above as for the ILA score [IPF transcripts]. We tested the association of each risk score with outcomes in the COPDGene testing set and ECLIPSE.

\section{Outcomes}

We examined two primary outcomes available in both cohorts: (1) prevalent ILA (at the time of blood sample collection), and (2) time-to-death. With respect to time-to-death, COPDGene participants were followed for up to 5 years after collection of RNA-seq data, and ECLIPSE participants for up to 8 years.

\section{Models and model specifications}

We used logistic regression to test the association of each of the 50 transcripts with ILA and compared the direction of effect for ILA to the transcript direction (up or down) in the IPF mortality score. We also compared the association of the 50 individual transcripts 
with all-cause mortality and compared the effect direction to both the effect for ILA association and direction in the original IPF mortality score. We assessed the association of each risk score (IPF score, ILA score [IPF transcripts], ILA score [all transcripts]) with ILA and time-to-death. Logistic regression was used to assess associations with ILA. We used Cox regression ${ }^{29}$ to test associations with time-to-death (survival R package ${ }^{30}$ ). Models were adjusted for age, sex, race, body-mass index, pack-years of cigarette smoking, and current smoking status (at the time of blood sample collection). For time-to-death analyses, we also performed stratified analyses in ILA and non-ILA participants. For ILA, the Bonferroni-adjusted threshold was $0.05 / 3$ risk scores $/ 2$ cohorts $=0.0083$. For time-todeath analyses, the Bonferroni-adjusted threshold was $0.05 / 3$ risk scores $/ 2$ cohorts $/ 3$ strata $=0.0028$. Proportional hazards assumptions were evaluated with Schoenfeld residual plots and tests. As a sensitivity analysis, we further adjusted models for white blood cell differential counts to assess whether white cell counts attenuated the observed signals. To combine the signals between the two testing cohorts (COPDGene testing, and ECLIPSE), we performed fixed-effects inverse variance-weighted meta-analyses using the meta $\mathrm{R}$ package ${ }^{31}$.

\section{Mediation analyses}

We noted that the associations of the ILA score [IPF transcripts] with time-to-death were substantially attenuated when age was added to models. Therefore, we performed causal mediation analyses to determine whether the effects of age on mortality were mediated through this score. We used the medflex R package ${ }^{32}$ to perform natural effects causal mediation analyses ${ }^{33,34}$ in the COPDGene testing set. We considered age as the exposure, and death (binary) as the outcome in logistic regression analyses. We considered the ILA score [IPF transcripts] as the mediator, and a p-value for the natural indirect effects less than 0.05 was considered significant.

\section{Characterization of IPF and ILA score genes}

To gain biologic insight into the relationship between genes that comprised the risk scores, we examined Pearson correlation coefficients between each of the 50 transcripts and constructed a heatmap of correlation coefficients. The observed correlation structure allowed us to use the sigora $\mathrm{R}$ package ${ }^{35}$ to perform pathway enrichment analyses for the 50 gene transcripts. We also evaluated how changing the number of genes in the ILA score [IPF transcripts] affected predictive performance (Supplementary Methods).

All analyses were performed in R version 4.0.3 (www.r-project.org). Normality for continuous variables was assessed by visual inspection of histograms. Results were reported as mean \pm standard deviation or median [interquartile range], as appropriate. Continuous variables were compared with Student t-tests or Wilcoxon tests, and categorical variables were compared with analysis of variance (ANOVA) or Kruskal-Wallis tests, as appropriate. A p-value less than 0.05 was considered nominally significant, and pvalues below Bonferroni-adjusted thresholds were considered significant. 


\section{Results}

Characteristics of study participants

Figure 1 is a schematic of the study design. We included 1,469 COPDGene participants from the 5-year follow up visit with RNA-seq and visual scoring of ILA phenotype data, and 571 ECLIPSE participants with microarray and ILA phenotype data. Table 1 shows demographic characteristics and outcomes in the COPDGene training set $(n=734)$, COPDGene testing set ( $n=735)$, and ECLIPSE $(\mathrm{n}=571)$. Characteristics were similar across COPDGene training and testing sets. Compared to COPDGene, ECLIPSE participants were more likely to be male, were all European ancestry, had more pack-years of smoking, were less likely to be current smokers, were less likely to have ILA, and had a higher proportion of deaths.

\section{Development of risk scores}

Fifty out of the 52 genes from Herazo-Maya et al. ${ }^{14,15}$ were available in both cohorts (C2ORF27A and SNHG1 were missing). First, using these 50 genes, we calculated the IPF score in COPDGene and ECLIPSE participants. We noted that when testing the associations of individual transcripts with ILA or death, 23 genes had a discordant direction of effect for ILA and 3 genes (HLA-DP1, $H L A-D P 2, L P A R 6)$ had a discordant direction of effect for all-cause mortality compared to the directions of effects reported for the IPF score (Table E1). Second, we used the 50 available genes to construct a LASSO penalized regression model in the COPDGene training set, which was optimized to the outcome of ILA (ILA score [IPF transcripts]). Table E2 shows the ILA score [IPF transcripts] gene transcripts, beta coefficients and comparisons of directions of effects to the IPF risk score. Only three transcripts ( $L B H, G B P 4$, $B T N 3 A 1)$ were significantly associated with ILA, all of which were included in the ILA score [IPF transcripts]. The ILA score [IPF transcripts] included 11 transcripts (BTN3A1, CPED1, CXCR6, GBP4, GPR174, IL7R, LBH, LPAR6, LRRC39, NAP1L2, PLBD1), six of which had a discordant direction of effect with the IPF score (BTN3A1, CPED1, GBP4, GPR174, LPAR6, NAP1L2). The distribution of the ILA score [IPF transcripts] in both cohorts is shown in Figure E1. We then plotted range-standardized predicted probabilities in the COPDGene testing set and observed overlapping but distinct distributions between the two risk scores (Figure E2). We additionally used genome-wide transcripts to train a risk score to ILA (ILA score [all transcripts]); this score included 25 transcripts (Table E3), none of which overlapped with the 50 genes in the IPF score. We then tested these risk scores (IPF score, ILA score [IPF transcripts], ILA score [all transcripts]) for association with ILA and time-to-death in the COPDGene testing set and ECLIPSE.

Association of risk scores with ILA

The IPF score was not associated with ILA in the COPDGene testing set or ECLIPSE (Table 2). The univariable associations of the ILA score [IPF transcripts] with ILA in both cohorts is shown in Figure 2A. In multivariable models (Table 2), the ILA score [IPF 
transcripts] was associated with ILA in the COPDGene testing set and ECLIPSE (meta-analysis OR 1.4 [95\% CI: 1.2-1.6], p=6.8e-5). As a sensitivity analysis, we further adjusted models for white blood cell counts, and observed similar results (Table E4).

Association of risk scores with time-to-death

The IPF risk score was not associated with mortality in the COPDGene testing set or ECLIPSE (Table 3). The univariable associations of the ILA score [IPF transcripts] with death in both cohorts is shown in Figure 2B. In multivariable analyses (Table 3), the ILA score [IPF transcripts] is associated with time-to-death in all participants, but not the ILA participants. Meta-analyses demonstrate a significant association of the ILA score [IPF transcripts] with time-to-death (Figure 3) in all (meta-analysis HR 1.25 [95\% CI: 1.12-1.41], $\mathrm{p}=1.25 \mathrm{e}-4$ ) and non-ILA (meta-analysis HR 1.33 [95\% CI: 1.17-1.52], p=2.17e-5) participants. As a sensitivity analysis, we further adjusted models for white blood cell counts and observed similar results (Table E5). In causal mediation analyses, we observed that the ILA score [IPF transcripts] mediated the effects of age on mortality (natural indirect effect $p=0.003$; proportion mediated $=11.8 \%$ [95\% CI: $4.04 \%-22.6 \%]$ ).

We examined the ILA score [all transcripts] for association with ILA and all-cause mortality. We found that this new score was associated with ILA and all-cause mortality in the COPDGene testing set, but only with all-cause mortality in ECLIPSE (Table E6).

\section{Characterization of the gene signature}

Having demonstrated that the genes in the IPF mortality score can be re-weighted to create an ILA score, and that this ILA score [IPF transcripts] consistently associates with ILA and time-to-death in two cohorts, we sought to understand how biological processes annotated to the IPF mortality genes relate to our outcomes. We observed that these 50 genes are highly correlated with each other and may represent coordinated biological processes. A heatmap of Pearson correlation coefficients is shown in Figure E3. In pathway enrichment analyses, the $50 \mathrm{IPF}$ score genes were associated with three pathways: Butyrophilin family interactions, P2Y receptors, and immunoregulatory interactions between lymphoid and non-lymphoid cells (Table E7). The effects of varying levels of genes in the ILA score [IPF transcripts] on predictive performance for ILA are shown in the supplement (Supplementary Results; Table E8; Figure E4).

\section{Discussion}

While there has been extensive research into gene expression changes associated with IPF $^{36-39}$, no studies, to our knowledge, have examined gene expression profiles in ILA. ILA may progress to pulmonary fibrosis in certain instances ${ }^{4,5}$, and IPF and ILA have demonstrated overlapping yet distinct genetic underpinnings ${ }^{10}$. In this study of over 2,000 individuals with blood gene expression and ILA phenotype data, we examined the association of a blood IPF gene expression mortality score with ILA and all-cause mortality. We found that a previously described IPF score was not associated with ILA or mortality. By applying penalized regression to the IPF 
gene transcripts, we developed an 11-transcript ILA score. Six of these genes had discordant directions of effects compared to the IPF score, which may implicate important mechanisms regulating whether an individual develops ILA versus progressing to pulmonary fibrosis. While there may be some shared pathogenic mechanisms between ILA and IPF, the amount of discordance we observed further suggests that some of those with ILA (among populations of smokers) are likely distinct from IPF. Two ILA scores, derived from IPF score or genome-wide transcripts, were associated with all-cause mortality in both cohorts, suggesting that the transcripts relevant to ILA risk may represent general risk factors for mortality. We identified a peripheral blood signature of ILA, demonstrated overlapping and distinct gene transcripts between ILA and IPF, and lend insight into how gene expression profiles and biological pathways associated with IPF prognosis relate to ILA and all-cause mortality.

Our analyses may lend insight into biological processes relevant to ILA. We developed our risk score using LASSO, a method that reduces collinearity amongst features and optimizes prediction accuracy, but which does not necessarily choose the most biologically relevant features; thus, biological interpretation must be performed with caution. Despite this caveat, many of the genes identified as being predictive of ILA have been implicated in IPF pathogenesis, and the correlation structure amongst IPF risk score genes allowed us to perform pathway enrichment analyses and link the predictive transcripts to specific biological processes.

Five genes demonstrated concordant directions of effects between the ILA [IPF transcripts] and IPF scores $(C X C R 6, I L 7 R, L B H$, $L R R C 39, P L B D 1)$, suggesting these genes may represent important biologic processes in promoting the progression of pulmonary fibrosis. $I L 7 R$ had the largest absolute effect size in the ILA score [IPF transcripts] and was inversely associated with ILA. Our results are consistent with in vitro and in vivo molecular evidence demonstrating that IL-7 inhibits fibroblast TGF- $\beta$ production in pulmonary fibrosis ${ }^{40}$. CXCR6 is a chemokine receptor that attracts T cells to the lungs ${ }^{41}$ and can promote the epithelial-mesenchymal transition in cell lines ${ }^{42}$, an event that may be important to the progression of pulmonary fibrosis. $L B H$ is a transcription factor involved in Wnt signaling and is highly expressed in sub-populations of matrix fibroblasts from mouse lung ${ }^{43}$. Thus, there is already evidence that $I L 7 R, L B H$, and $C X C R 6$ are important for understanding processes that promote pulmonary fibrosis, and $L R R C 39$ and $P L B D 1$ are additional targets for future studies.

The transcripts with discordant directions of effects compared to the IPF score (BTN3A1, GBP4, CPED1, GPR174, LPAR6, NAP1L2) support the notion that ILA may represent a collection of disorders, some of which are distinct from IPF. This observation is consistent with genetic analyses of IPF and ILA. A genome-wide association study (GWAS) of ILA identified four genome-wide significant variants (in FCF1P3, IPO11, HTR1E, MUC5B); while the MUC5B rs35705950 promoter polymorphism is well known in IPF, the other three loci were not associated with IPF. Similarly, out 12 previously reported IPF GWAS variants, only four other variants were significantly associated with ILA (DPP9, DSP, FAM13A, IVD), though most of the others were consistent in effect 
direction. Whether the genetic variants uniquely associated with ILA indirectly regulate the discordant genes identified in the current study is unclear and requires further investigation.

The transcripts with discordant directions of effects between the ILA [IPF transcripts] and IPF scores allude to divergent biologic processes that could play a role in determining whether a person develops ILA or progresses to irreversible fibrosis. BTN3A1 is part of the Butyrophilin immunoglobulin superfamily. Butyrophilins may play a role in facilitating interactions between adaptive and innate immune cells ${ }^{44}$. GBP4 is a guanylate binding protein that facilitates second messenger signaling for interferons, and has been observed to increase in response to cytokine stimulation in IPF lungs ${ }^{45}$. Genetic variation in GPR174 is associated with susceptibility to autoimmune disease, and GPR174-deficient mice were resistant to lipopolysaccharide-induced cytokine storm ${ }^{46}$. NAP1L2 promotes histone acetylation during neuronal differentiation ${ }^{47}$ and other members of the same protein family have demonstrated increased expression in lung fibroblasts from IPF patients ${ }^{48}$. Taken together, these data suggest that immune responses to environmental/infectious insults, genetic variation, and epigenetic modifications may be important to understanding the distinct pathogenic mechanisms present in ILA that may lead to IPF.

We observed that the ILA score [IPF transcripts] was associated with all-cause mortality, but that this association was driven by nonILA participants, albeit with small samples sizes for ILA. In single gene association analyses for the 50 IPF score genes, nearly half of all transcripts had discordant effect directions for ILA association compared to associations with all-cause mortality and in comparison to gene weights in the IPF score. Conversely, the 50 IPF gene effect directions in association with all-cause mortality (in all study participants) were highly concordant with the directions of weights in the IPF score with only three genes having discordant effect directions. Further, an ILA score trained using genome-wide transcripts in the COPDGene training data was not associated with ILA in both the COPDGene testing set and ECLIPSE but was associated with all-cause mortality in both cohorts. These data suggest that transcripts associated with ILA risk may also be risk factors for all-cause mortality. When training a gene expression model to ILA, we identified a gene set associated with all-cause mortality; however, an ILA score derived from the limited set of IPF score genes is associated with ILA and mortality. These observations suggest that the IPF score represents a mixture of transcripts relevant to all-cause mortality and the ILA-IPF axis.

LPAR6 was the only non-HLA transcript with concordant directions of effects when tested for association with ILA and all-cause mortality and had discordant directions of effects compared to the IPF score. LPAR6 is a lysophosphatidic acid (LPA) receptor. LPA has been shown to signal through its G-protein-coupled receptors to induce pro-inflammatory signals from stressed epithelial cells and activate TGF signaling ${ }^{49}$. Thus, LPAR6 could represent a shared mechanism between ILA and all-cause mortality or it could be on the causal pathway between ILA and death. 
As aging is a major driver of mortality, we sought to determine whether the effects of the ILA score [IPF transcripts] on mortality represent an aging effect. In causal mediation analyses, we found that about $12 \%$ of the effect of age on mortality was mediated through the ILA score [IPF transcripts]. Thus, the 11 genes in the ILA score [IPF transcripts] appear to be important for overall mortality and partially represent aging effects on mortality. However, the majority of aging effects are not captured by the ILA score [IPF transcripts], which highlights the need for further research into the shared and divergent biological processes related to ILA, aging, and mortality.

Strengths of this study include replication in two well-characterized cohorts of smokers, cross-technology replication (both RNA-seq and microarray), and the application of causal inference analyses to examine the relationship between IPF and ILA transcriptomic risk with aging and risk of death. However, we were not able to assess the association of the ILA risk scores with the development or progression of ILA due to data availability. Our primary analysis was of genes identified in a study of IPF, which allowed an assessment of the contrasts between IPF mortality and ILA risk in a well-validated set of genes. Notably, attempting to use a larger set of genes in the blood transcriptome, including other transcripts that may be more predictive of ILA risk, was not superior. Other approaches using blood gene expression and additional -Omics data (such as methylation, proteomic, microbiome) may allow us to delve deeper into the mechanisms underlying our observed associations, as well as longitudinal studies of ILA and IPF.

In conclusion, a peripheral blood gene signature associated with IPF mortality was not associated with ILA or mortality in two wellcharacterized cohorts of smokers. An ILA gene expression score, derived from the genes in the IPF score, was reproducibly associated with ILA and all-cause mortality in current and former smokers. Separately, an ILA score derived from genome-wide transcripts was associated with all-cause mortality, but not ILA in validation studies. Approximately half of the genes in the ILA score [IPF transcripts] were of opposite direction in the IPF score, and genes associated with ILA may also be risk factors for mortality and partially represent aging effects on mortality. Genes identified in this study may be important candidates to further examine in the pathogenesis and progression of ILA to IPF and in mortality. 


\section{References}

1 Washko GR, Hunninghake GM, Fernandez IE, et al. Lung volumes and emphysema in smokers with interstitial lung abnormalities. N Engl J Med 2011; 364: 897-906.

2 Hunninghake GM, Hatabu H, Okajima Y, et al. MUC5B promoter polymorphism and interstitial lung abnormalities. $N$ Engl $J$ Med 2013; 368: 2192-200.

3 Hatabu H, Hunninghake GM, Richeldi L, et al. Interstitial lung abnormalities detected incidentally on CT: a Position Paper from the Fleischner Society. Lancet Respir Med 2020; 8: 726-37.

4 Putman RK, Gudmundsson G, Axelsson GT, et al. Imaging Patterns Are Associated with Interstitial Lung Abnormality Progression and Mortality. Am J Respir Crit Care Med 2019; 200: 175-83.

$5 \quad$ Araki T, Putman RK, Hatabu H, et al. Development and Progression of Interstitial Lung Abnormalities in the Framingham Heart Study. Am J Respir Crit Care Med 2016; 194: 1514-22.

6 Doyle TJ, Washko GR, Fernandez IE, et al. Interstitial lung abnormalities and reduced exercise capacity. Am J Respir Crit Care Med 2012; 185: 756-62.

7 Lederer DJ, Enright PL, Kawut SM, et al. Cigarette smoking is associated with subclinical parenchymal lung disease: the Multi-Ethnic Study of Atherosclerosis (MESA)-lung study. Am J Respir Crit Care Med 2009; 180: 407-14.

8 Tsushima K, Sone S, Yoshikawa S, Yokoyama T, Suzuki T, Kubo K. The radiological patterns of interstitial change at an early phase: over a 4-year follow-up. Respir Med 2010; 104: 1712-21.

9 Putman RK, Hatabu H, Araki T, et al. Association Between Interstitial Lung Abnormalities and All-Cause Mortality. JAMA 2016; 315: 672-81.

10 Hobbs BD, Putman RK, Araki T, et al. Overlap of Genetic Risk between Interstitial Lung Abnormalities and Idiopathic Pulmonary Fibrosis. Am J Respir Crit Care Med 2019; 200: 1402-13.

11 Putman RK, Gudmundsson G, Araki T, et al. The MUC5B promoter polymorphism is associated with specific interstitial lung abnormality subtypes. Eur Respir J 2017; 50. DOI:10.1183/13993003.00537-2017.

12 Baumgartner KB, Samet JM, Stidley CA, Colby T V, Waldron JA. Cigarette smoking: a risk factor for idiopathic pulmonary fibrosis. Am J Respir Crit Care Med 1997; 155: 242-8.

13 Parker MM, Chase RP, Lamb A, et al. RNA sequencing identifies novel non-coding RNA and exon-specific effects associated with cigarette smoking. BMC Med Genomics 2017; 10: 58.

14 Herazo-Maya JD, Noth I, Duncan SR, et al. Peripheral blood mononuclear cell gene expression profiles predict poor outcome in idiopathic pulmonary fibrosis. Sci Transl Med 2013; 5: 205 ra136.

15 Herazo-Maya JD, Sun J, Molyneaux PL, et al. Validation of a 52-gene risk profile for outcome prediction in patients with idiopathic pulmonary fibrosis: an international, multicentre, cohort study. Lancet Respir Med 2017; 5: 857-68.

16 Regan EA, Hokanson JE, Murphy JR, et al. Genetic Epidemiology of COPD (COPDGene) Study Design. COPD J Chronic 
Obstr Pulm Dis 2011; 7: 32-43.

17 Vestbo J, Anderson W, Coxson HO, et al. Evaluation of COPD Longitudinally to Identify Predictive Surrogate End-points (ECLIPSE). Eur Respir J 2008; 31: 869-73.

18 Hatabu H, Hunninghake GM, Lynch DA. Interstitial Lung Abnormality: Recognition and Perspectives. Radiology 2019; 291: $1-3$.

19 Hata A, Schiebler ML, Lynch DA, Hatabu H. Interstitial Lung Abnormalities: State of the Art. Radiology $2021 ;$ : 204367.

20 Hino T, Lee KS, Han J, Hata A, Ishigami K, Hatabu H. Spectrum of Pulmonary Fibrosis from Interstitial Lung Abnormality to Usual Interstitial Pneumonia: Importance of Identification and Quantification of Traction Bronchiectasis in Patient Management. Korean J Radiol 2021; 22: 811-28.

21 Jiang H, Lei R, Ding S-W, Zhu S. Skewer: a fast and accurate adapter trimmer for next-generation sequencing paired-end reads. BMC Bioinformatics 2014; 15: 182.

22 DeLuca DS, Levin JZ, Sivachenko A, et al. RNA-SeQC: RNA-seq metrics for quality control and process optimization. Bioinformatics 2012; 28: 1530-2.

23 Dobin A, Gingeras TR. Mapping RNA-seq Reads with STAR. Curr Protoc Bioinforma 2015; 51: 11.14.1-11.14.19.

24 Ritchie ME, Phipson B, Wu D, et al. limma powers differential expression analyses for RNA-sequencing and microarray studies. Nucleic Acids Res 2015; 43: e47.

25 Obeidat M, Nie Y, Chen V, et al. Network-based analysis reveals novel gene signatures in peripheral blood of patients with chronic obstructive pulmonary disease. Respir Res 2017; 18: 72.

26 Carvalho BS, Irizarry RA. A framework for oligonucleotide microarray preprocessing. Bioinformatics 2010; 26: 2363-7.

27 Irizarry RA, Hobbs B, Collin F, et al. Exploration, normalization, and summaries of high density oligonucleotide array probe level data. Biostatistics 2003; 4: 249-64.

28 Hochreiter S, Clevert D-A, Obermayer K. A new summarization method for Affymetrix probe level data. Bioinformatics 2006; 22: $943-9$.

29 Cox DR. Regression models and life tables. J R Stat Soc Ser B 1972. DOI:10.1079/IVPt200454()IN.

30 T T. A Package for Survival Analysis in R. R package version 3.1-12. https://CRANR-project.org/package =survival 2020.

31 G S. meta: An R package for meta-analysis. R News 2007; 7: 40-5.

32 Johan Steen, Tom Loeys, Beatrijs Moerkerke SV. medflex: An R Package for Flexible Mediation Analysis using Natural Effect Models. J Stat Softw 2017; 76: 1-46.

33 Lange, T., Vansteelandt, S., \& Bekaert M. A Simple Unified Approach for Estimating Natural Direct and Indirect Effects. Am J Epidemiol 2012; 176: 190-5.

34 Vansteelandt, S., Bekaert, M., \& Lange T. Imputation Strategies for the Estimation of Natural Direct and Indirect Effects. Epidemiologic Methods. Epidemiol Method 2012; 1: Article 7.

35 Foroushani ABK, Brinkman FSL, Lynn DJ. Pathway-GPS and SIGORA: identifying relevant pathways based on the over- 
representation of their gene-pair signatures. PeerJ 2013; 1: e229.

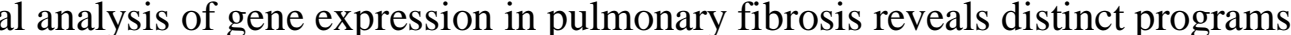
regulating lung inflammation and fibrosis. Proc Natl Acad Sci U S A 2000; 97: 1778-83.

37 Huang Y, Oldham JM, Ma S-F, et al. Blood Transcriptomic Predicts Progression of Pulmonary Fibrosis and Associates Natural Killer Cells. Am J Respir Crit Care Med 2021; published online March 9. DOI:10.1164/rccm.202008-3093OC.

38 DePianto DJ, Chandriani S, Abbas AR, et al. Heterogeneous gene expression signatures correspond to distinct lung pathologies and biomarkers of disease severity in idiopathic pulmonary fibrosis. Thorax 2015; 70: 48-56.

39 Reyfman PA, Walter JM, Joshi N, et al. Single-Cell Transcriptomic Analysis of Human Lung Provides Insights into the Pathobiology of Pulmonary Fibrosis. Am J Respir Crit Care Med 2019; 199: 1517-36.

40 Huang M, Sharma S, Zhu LX, et al. IL-7 inhibits fibroblast TGF-beta production and signaling in pulmonary fibrosis. $J$ Clin Invest 2002; 109: 931-7.

41 Morgan AJ, Guillen C, Symon FA, et al. Expression of CXCR6 and its ligand CXCL16 in the lung in health and disease. Clin Exp Allergy 2005; 35: 1572-80.

42 Ma Z, Ma C, Zhang Q, et al. Role of CXCL16 in BLM-induced epithelial-mesenchymal transition in human A549 cells. Respir Res 2021; 22: 42.

43 Xie T, Wang Y, Deng N, et al. Single-Cell Deconvolution of Fibroblast Heterogeneity in Mouse Pulmonary Fibrosis. Cell Rep 2018; 22: 3625-40.

44 Miles T, Hoyne GF, Knight DA, Fear MW, Mutsaers SE, Prêle CM. The contribution of animal models to understanding the role of the immune system in human idiopathic pulmonary fibrosis. Clin Transl Immunol 2020; 9: e1153.

45 Kass DJ, Yu G, Loh KS, et al. Cytokine-like factor 1 gene expression is enriched in idiopathic pulmonary fibrosis and drives the accumulation of CD4+ T cells in murine lungs: evidence for an antifibrotic role in bleomycin injury. Am J Pathol 2012; 180: $1963-78$.

46 Qiu D, Chu X, Hua L, et al. Gpr174-deficient regulatory T cells decrease cytokine storm in septic mice. Cell Death Dis 2019; 10: 233.

47 Attia M, Rachez C, De Pauw A, Avner P, Rogner UC. Nap112 promotes histone acetylation activity during neuronal differentiation. Mol Cell Biol 2007; 27: 6093-102.

48 Plantier L, Renaud H, Respaud R, Marchand-Adam S, Crestani B. Transcriptome of Cultured Lung Fibroblasts in Idiopathic Pulmonary Fibrosis: Meta-Analysis of Publically Available Microarray Datasets Reveals Repression of Inflammation and Immunity Pathways. Int J Mol Sci 2016; 17. DOI:10.3390/ijms17122091.

49 Ninou I, Magkrioti C, Aidinis V. Autotaxin in Pathophysiology and Pulmonary Fibrosis. Front Med 2018; 5: 180. 


\section{Table legends}

Table 1: Characteristics of study participants. ILA=interstitial lung abnormalities. *ILA prevalence in the overall population was $5.7 \%$ $(586 / 10,364)$ in COPDGene and $1.3 \%(37 / 2,746)$ in ECLIPSE.

Table 2: Odds ratios of the IPF and ILA [IPF transcripts] risk scores with ILA in the COPDGene test set (n=735) and ECLIPSE $(\mathrm{n}=571)$. Logistic regression models were adjusted for age, sex, race, body-mass index, pack-years of smoking, and current smoking status. Inverse variance fixed-effects meta-analyses of the adjusted estimates were performed. ILA=interstitial lung abnormalities. $\mathrm{IPF}=\mathrm{idiopathic} \mathrm{pulmonary} \mathrm{fibrosis.}$

Table 3: Association of the IPF and ILA [IPF transcripts] scores with time-to-death in the COPDGene test set ( $\mathrm{n}=735$ ) and ECLIPSE $(\mathrm{n}=571)$. Multivariable Cox regression models were adjusted for age, sex, race, body-mass index, pack-years of smoking, and current smoking status. ILA = interstitial lung abnormalities.

\section{Figure legends}

Figure 1: Schematic of study design. ILA = interstitial lung abnormalities. IPF = idiopathic pulmonary fibrosis. LASSO = least absolute shrinkage and selection operator.

Figure 2: Violin and box plots showing the unadjusted association of the ILA score [IPF transcripts] with ILA (A) and death (B) in the COPDGene test set $(n=735)$ and ECLIPSE $(n=571)$.

Figure 3: Forest plots based on time-to-death analyses of the ILA score [IPF transcripts] in the COPDGene test set ( $\mathrm{n}=735$ ) and ECLIPSE ( $\mathrm{n}=571)$. Cox proportional hazards models were fit, adjusting for age, sex, race, body-mass index, pack-years of smoking, and current smoking. Hazard ratios are shown as per standard deviation increase in the ILA score. ILA=interstitial lung abnormalities. 
Table 1: Characteristics of study participants. ILA=interstitial lung abnormalities. *ILA prevalence in the overall population was $5.7 \%$ $(586 / 10,364)$ in COPDGene and 1.3\% $(37 / 2,746)$ in ECLIPSE.

\begin{tabular}{|c|c|c|c|c|}
\hline Characteristics & COPDGene training sample & COPDGene testing sample & ECLIPSE & $p$ \\
\hline $\mathrm{n}$ & 734 & 735 & 571 & \\
\hline Age in years (mean (SD)) & $65.08(8.53)$ & $64.91(8.60)$ & $64.04(6.10)$ & 0.054 \\
\hline Sex (No. $(\%)$ female) & $341(46.5)$ & $371(50.5)$ & $195(34.2)$ & $<0.001$ \\
\hline Race (No. (\%) African American) & $178(24.3)$ & $171(23.3)$ & $0(0.0)$ & $<0.001$ \\
\hline Pack-years of smoking (mean (SD)) & $43.71(23.86)$ & $42.38(21.85)$ & $47.27(26.45)$ & 0.002 \\
\hline Current smoking status (No. (\%)) & $253(34.5)$ & $250(34.1)$ & $129(22.6)$ & $<0.001$ \\
\hline \multicolumn{5}{|l|}{ Outcomes } \\
\hline ILA (No. $(\%)) *$ & $122(16.6)$ & $126(17.1)$ & $37(6.5)$ & $<0.001$ \\
\hline Dead (No. $(\%))$ & $84(11.4)$ & $73(9.9)$ & $107(30.7)$ & $<0.001$ \\
\hline Days followed (median [IQR]) & $1881.00[1603.75,2104.00]$ & $1916.50[1631.50,2140.75]$ & $2848.00[1425.00,2926.00]$ & $<0.001$ \\
\hline
\end{tabular}


Table 2: Odds ratios of the IPF and ILA [IPF transcripts] risk scores with ILA in the COPDGene test set (n=735) and ECLIPSE $(\mathrm{n}=571)$. Logistic regression models were adjusted for age, sex, race, body-mass index, pack-years of smoking, and current smoking status. Inverse variance fixed-effects meta-analyses of the adjusted estimates were performed. ILA=interstitial lung abnormalities. $\mathrm{IPF}=\mathrm{idiopathic} \mathrm{pulmonary} \mathrm{fibrosis.}$

\begin{tabular}{|c|c|c|c|c|c|c|c|c|c|c|}
\hline \multirow{3}{*}{ Score } & \multicolumn{4}{|c|}{ COPDGene } & \multicolumn{4}{|c|}{ ECLIPSE } & \multirow{2}{*}{\multicolumn{2}{|c|}{$\begin{array}{l}\text { Combined (Meta- } \\
\text { analyses) }\end{array}$}} \\
\hline & Unadjusted & & Adjusted & & Unadjusted & & Adjusted & & & \\
\hline & OR $(95 \% C I)$ & $p$ & OR $(95 \% C I)$ & $p$ & OR $(95 \% C I)$ & $p$ & OR $(95 \% C I)$ & $p$ & OR $(95 \% C I)$ & $p$ \\
\hline \multirow{2}{*}{$\begin{array}{l}\text { IPF score } \\
\text { ILA score [IPF } \\
\text { transcripts] }\end{array}$} & $1.8(1.1-2.8)$ & 0.018 & $1.7(1.1-2.8)$ & 0.023 & $0.64(0.22-1.9)$ & 0.41 & $0.63(0.22-1.8)$ & 0.4 & $1.5(0.95-2.3)$ & 0.083 \\
\hline & $1.4(1.2-1.7)$ & $\begin{array}{c}6.70 \mathrm{E}- \\
05\end{array}$ & $1.3(1.1-1.6)$ & 0.0019 & $1.5(1.2-2)$ & 0.001 & $1.4(1.1-1.9)$ & 0.011 & $1.4(1.2-1.6)$ & $\begin{array}{c}6.80 \mathrm{E}- \\
05\end{array}$ \\
\hline
\end{tabular}


Table 3: Association of the IPF and ILA [IPF transcripts] scores with time-to-death in the COPDGene test set ( $\mathrm{n}=735$ ) and ECLIPSE $(\mathrm{n}=571)$. Multivariable Cox regression models were adjusted for age, sex, race, body-mass index, pack-years of smoking, and current smoking status. ILA = interstitial lung abnormalities.

\begin{tabular}{|c|c|c|c|c|c|c|}
\hline \multirow{2}{*}{ Score } & \multirow{2}{*}{ Cohort } & \multirow{2}{*}{ Stratum } & \multicolumn{2}{|c|}{ Unadjusted } & \multicolumn{2}{|c|}{ Adjusted } \\
\hline & & & $\mathrm{HR}(95 \% \mathrm{CI})$ & $\mathrm{p}$ & $\mathrm{HR}(95 \% \mathrm{CI})$ & $\mathrm{p}$ \\
\hline \multirow{6}{*}{ IPF score } & \multirow{3}{*}{ COPDGene test set } & All & $1.3(0.71-2.3)$ & 0.41 & $1.3(0.7-2.3)$ & 0.44 \\
\hline & & ILA & $1.3(0.47-3.8)$ & 0.6 & $1.2(0.44-3.6)$ & 0.68 \\
\hline & & non-ILA & $1.2(0.57-2.4)$ & 0.67 & $1.2(0.58-2.4)$ & 0.63 \\
\hline & \multirow{3}{*}{ ECLIPSE } & All & $1.3(0.77-2.1)$ & 0.36 & $1.2(0.75-2)$ & 0.4 \\
\hline & & ILA & $2.2(0.49-10)$ & 0.3 & $2.6(0.54-13)$ & 0.23 \\
\hline & & non-ILA & $1.2(0.72-2.1)$ & 0.47 & $1.2(0.7-2)$ & 0.54 \\
\hline \multirow{6}{*}{ ILA score [IPF transcripts] } & \multirow{3}{*}{ COPDGene test set } & All & $1.4(1.2-1.7)$ & $3.60 \mathrm{E}-05$ & $1.3(1.1-1.6)$ & 0.0012 \\
\hline & & ILA & $1(0.64-1.6)$ & 0.99 & $0.9(0.53-1.5)$ & 0.69 \\
\hline & & non-ILA & $1.6(1.3-2)$ & $2.50 \mathrm{E}-06$ & $1.6(1.3-2)$ & 4.00E-05 \\
\hline & \multirow{3}{*}{ ECLIPSE } & All & $1.3(1.1-1.4)$ & 0.003 & $1.2(1-1.4)$ & 0.023 \\
\hline & & ILA & $1.2(0.78-1.7)$ & 0.47 & $1.1(0.67-1.7)$ & 0.82 \\
\hline & & non-ILA & $1.3(1.1-1.5)$ & 0.006 & $1.2(1-1.4)$ & 0.027 \\
\hline
\end{tabular}




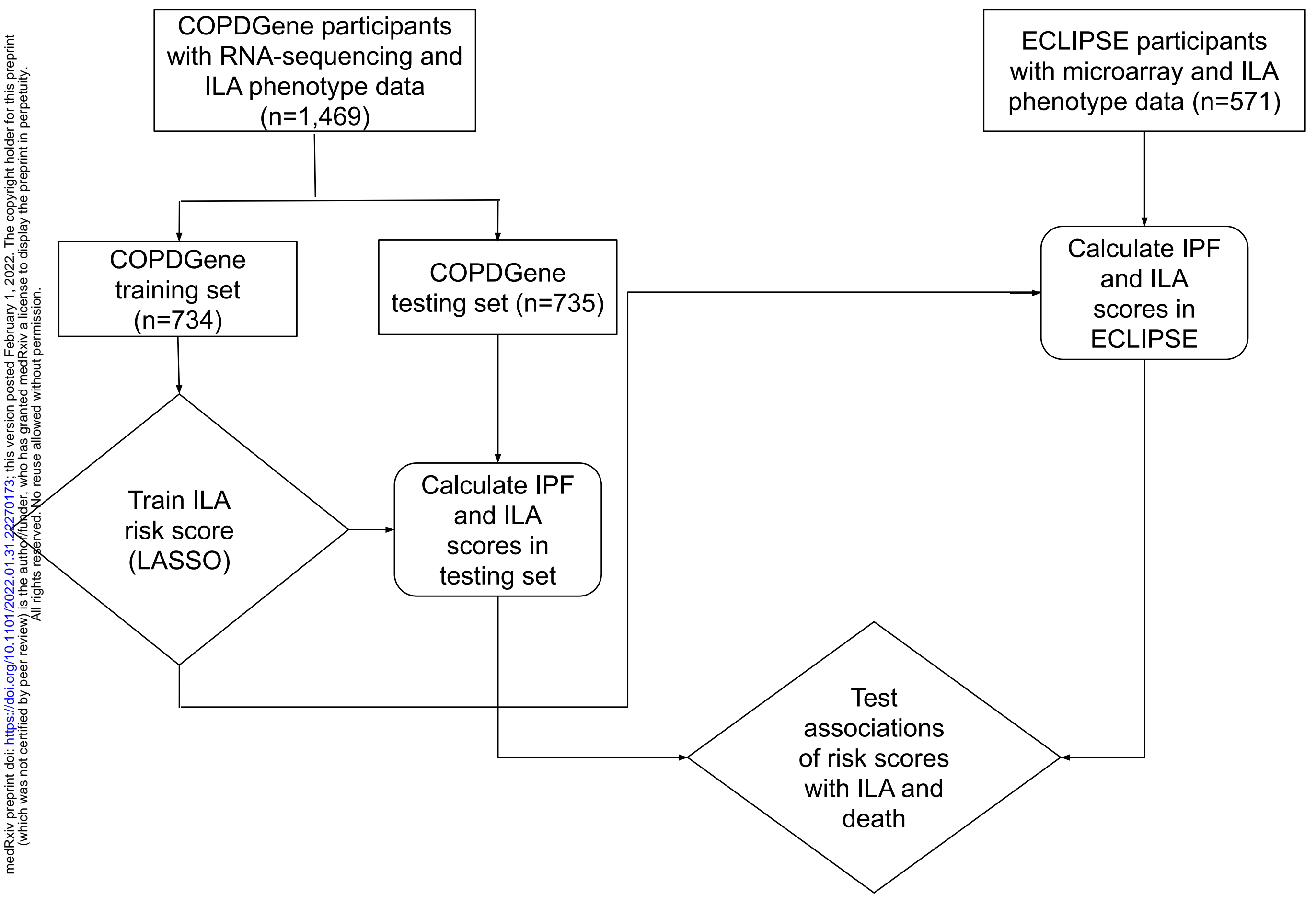




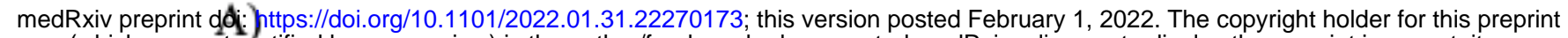
(which was not certified by peer review) is the author/funder, who has granted medRxiv a license to display the preprint in perpetuity.

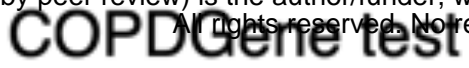
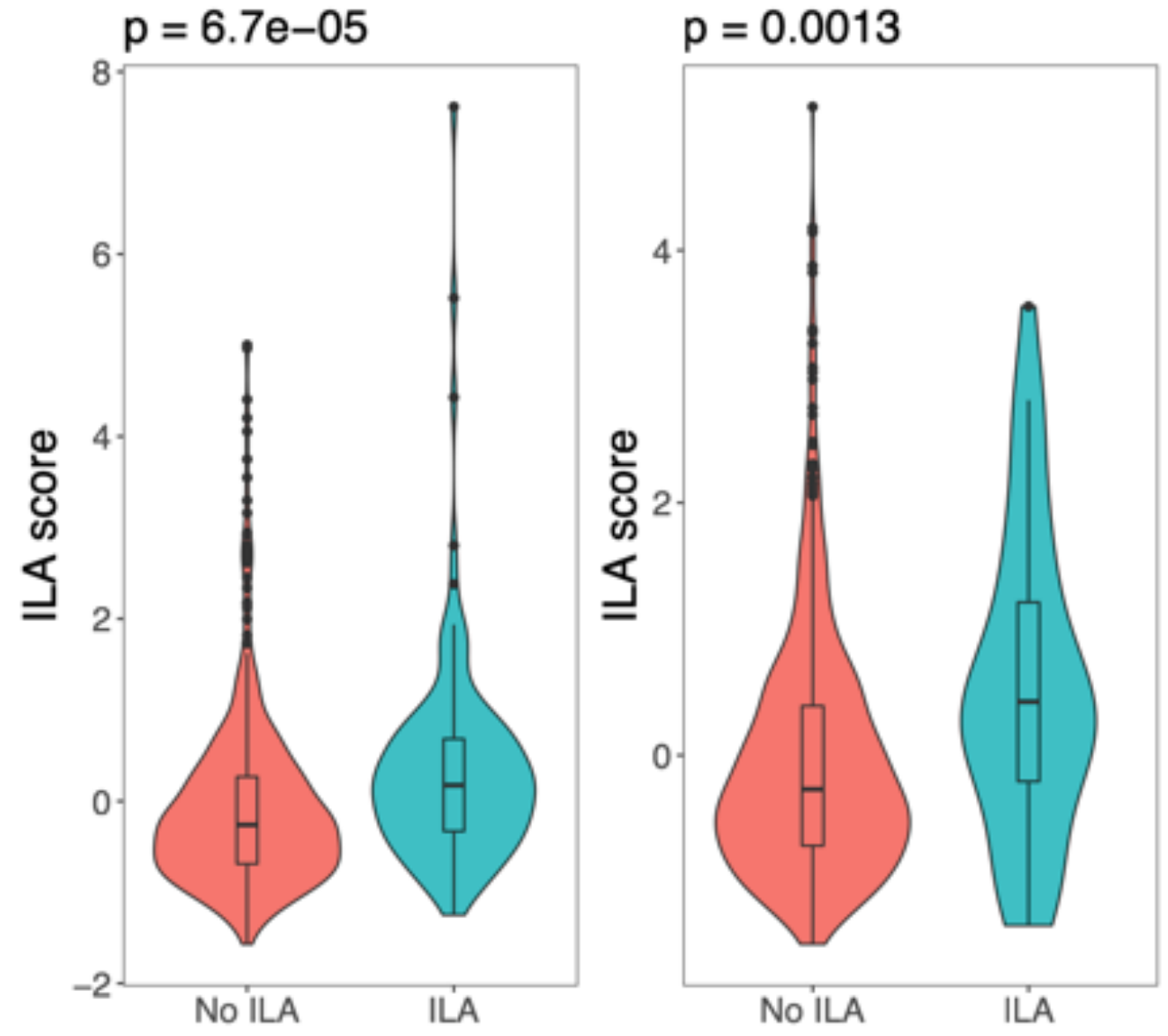

B)
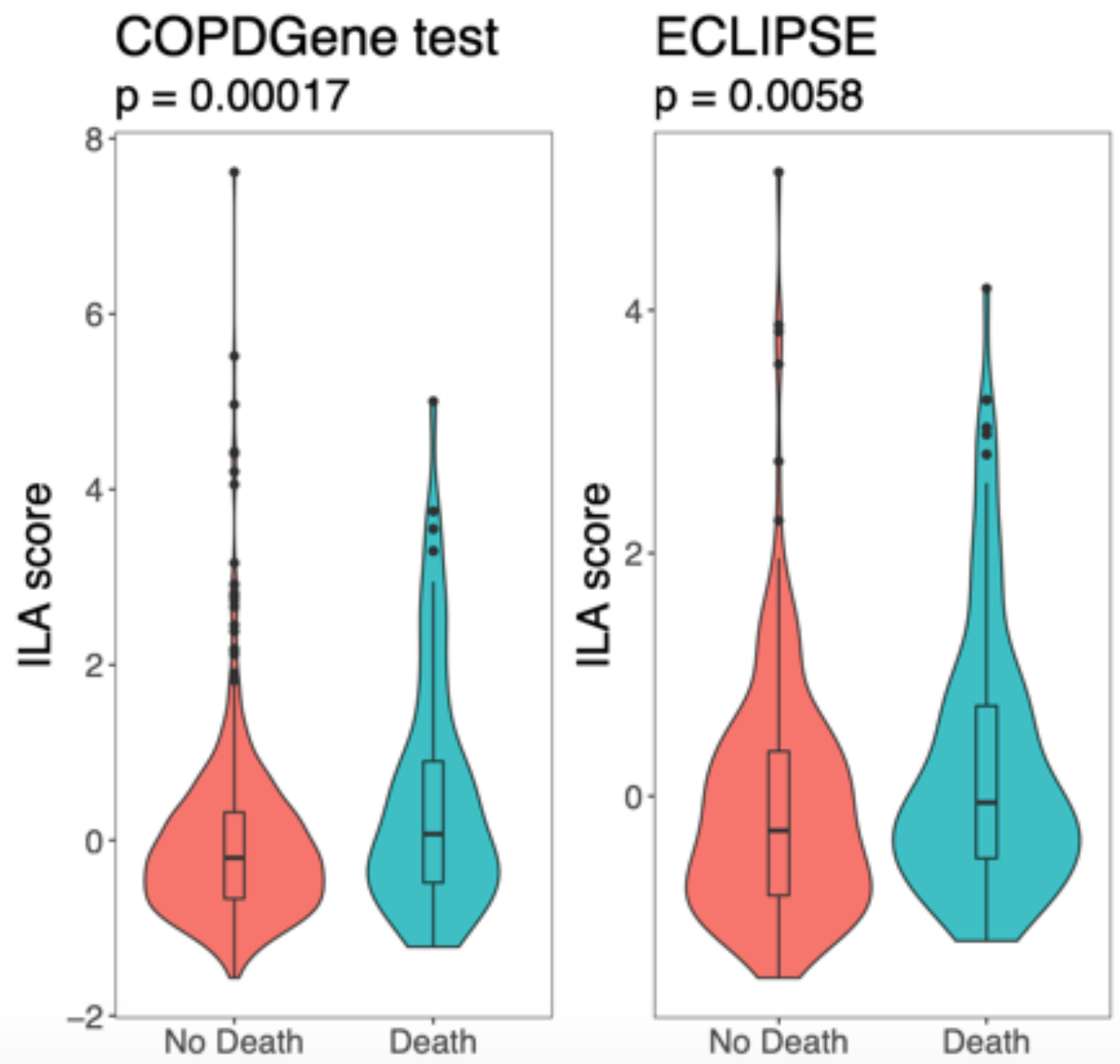
All

COPDGene

ECLIPSE

Fixed effect model

ILA

COPDGene

ECLIPSE

Fixed effect model

non-ILA

COPDGene

ECLIPSE

Fixed effect model

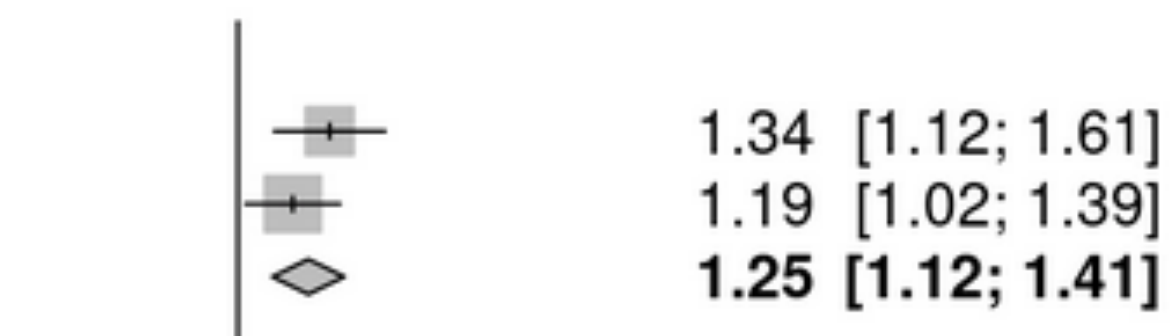

$0.90 \quad[0.53 ; 1.53]$ $1.05[0.67 ; 1.65]$ $0.99[0.70 ; 1.39]$
$1.58 \quad[1.27 ; 1.96]$

$1.20[1.02 ; 1.42]$

1.33 [1.17; 1.52]
0.25
0.5
1
2 